\title{
PARAMETRIC FOUR-PHOTON GENERATION OF PICOSECOND LIGHT AT HIGH CONVERSION EFFICIENCY
}

\author{
A. PENZKOFER, A. SEILMEIER and W. KAISER \\ Physik-Department der Technischen Universität München, Munich, Fed. Rep. Germany
}

Received 16 April 1975

\begin{abstract}
Parametric four-photon interaction in isotropic media was studied in the saturation range. Up to $10 \%$ of input laser energy could be converted into a broad frequency spectrum ranging from the ultraviolet to the infrared. Parameters which influence the conversion efficiency are discussed.
\end{abstract}

With picosecond light pulses of high peak intensity superbroad light spectra were observed in isotrupic media [1-4]. It has been shown in ref. [4] that such light spectra are generated by stimulated parametric four-photon interactions due to the resonant structure of the nonlinear susceptibility $\chi^{(3)}$. The continuous spectra ranging from the ultravislet to the infrared region present an interesting light source for picosecond spectroscopy.

In this letter we present new data of the parametric light generation in various isotropic media in the saturation range. The studies are extended to the infrared region. Calculations are presented which give a qualitative explanation of the experimental results.

In our experiments we used a mode-locked $\mathrm{Nd}-$ glass laser. A single light pulse was selected from the pulse train with an electro-optic shutter. The pulses were amplified to an energy value of approximately $5 \mathrm{~mJ}$ in a laser amplifier. The pulse duration was $\Delta t$ $\simeq 6 \mathrm{ps}$. The pulses were nearly bandwid th limited $(\Delta \tilde{\nu} \Delta t \simeq 0.6$ ). The intensity of the light pulses at the samples was varied with filters and lenses. The following investigations were performed:

1). The spectral distribution of the parametrically generated light was measured. At high input laser intensities a fairly smooth spectrum is observed which extends from the onsel of electronic transitions in the ultraviolet to the vibrational absorption bands in the infrared region. After a rapid exponential rise around $I_{\mathrm{L}} \simeq 5 \times 10^{10} \mathrm{~W} / \mathrm{cm}^{2}[4]$ the parametric light begins to saturate for larger input intensities. The spectral distribution of the generated light was measured with monochromators in conjunction with photomultipliers. (Full detection angle $\simeq 0.2 \mathrm{rad}$ in the forward direction). Measurements at the Stokes side of the laser frequency $\left(\widetilde{\nu}_{\mathrm{L}}=9455 \mathrm{~cm}^{-1}\right)$ were carried out with interference filters and $\mathrm{PbS}$ detectors. In fig. 1 the energy conversion per wavenumber of laser light into parametric light versus fre-

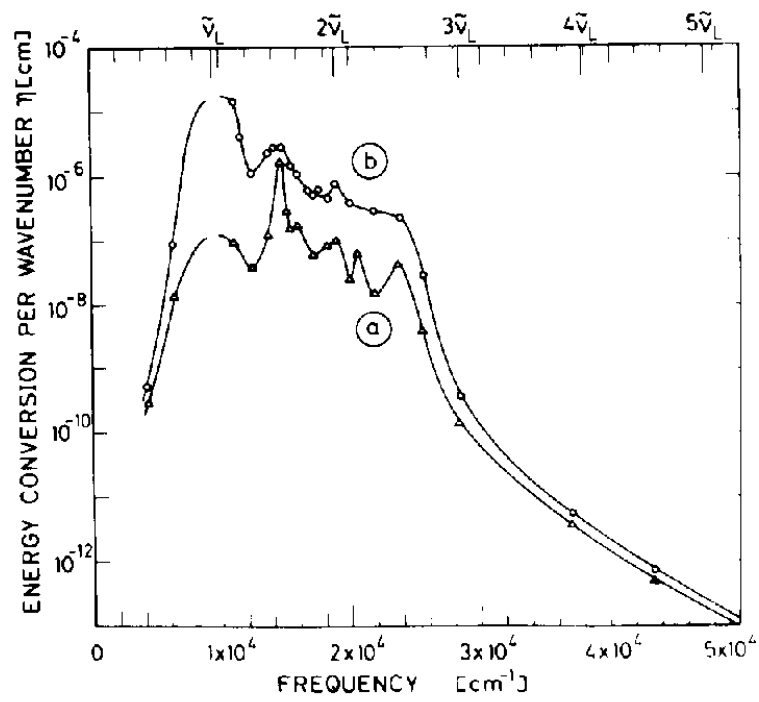

Fig. 1. Spectral distribution of parametric light in water ( $2 \mathrm{~cm}$ length) at a laser intensity of (a) $1 \times 10^{11} \mathrm{~W} / \mathrm{cm}^{2}$ and (b) $5 \times 10^{11} \mathrm{~W} / \mathrm{cm}^{2}$. 
quency is shown for the case of $\mathrm{H}_{2} \mathrm{O}$. (Cell length $l=2 \mathrm{~cm}$ ). The two curves were taken at input peak intensities of $1 \times 10^{11} \mathrm{~W} / \mathrm{cm}^{2}$ and $5 \times 10^{11} \mathrm{~W} / \mathrm{cm}^{2}$. The curves indicate that the energy conversion is largest between $\widetilde{\nu}_{\mathrm{L}}$ and $2 \widetilde{\nu}_{\mathrm{L}}$ with strong fall-off at higher and lower frequencies. The parametric light in the ultraviolet region (near the electronic absorption bands) is down by approximately six orders of magnitude compared to the values around $2 \widetilde{v}_{L}$. In the primary parametric four photon process, $\omega_{L}+\omega_{L} \rightarrow \omega_{3}+\omega_{4}$, signal light at $\omega_{3}$ is generated between $\omega_{L}$ and $2 \omega_{L}$. At frequencies exceeding $2 \widetilde{\nu}_{\mathrm{L}}$, light is generated by frequency conversion $\omega_{\mathrm{L}}+\omega_{\mathrm{L}}+\omega_{3}^{\prime} \rightarrow \omega_{4}^{\prime}$ and by higher order parametric four-photon processes $\omega_{L}+\omega_{3}^{\prime} \rightarrow \omega_{3}^{\prime \prime}+\omega_{4}^{\prime \prime}$ $[4,5] . \omega_{3}^{\prime}$ represents the frequency of an electromagnetic wave which was generated in the primary or following parametric process. The frequency conversion process becomes less efficient in the ultraviolet region because of the increased phase mismatch [5. Furthermore the efficiency of the parametric four-photon processes is reduced with increasing order in the ultraviolet region. The smaller parametric light generation in the infrared will be discussed later.

2). The total energy conversion of laser light into parametric light was determined for several substances by integrating spectral distribution curves such as those of fig. 1. In fig. 2a the total energy conversion $\eta_{\text {tot }}$ is shown for $\mathrm{H}_{2} \mathrm{O}$ (length $l=2 \mathrm{~cm}$ ) as a function of input peak intensity. An energy conversion up to $10 \%$ was observed. Within a factor of two, similar curves were obtained for $\mathrm{D}_{2} \mathrm{O}(l=2 \mathrm{~cm})$, quartz glass (Infrasil, $l=3 \mathrm{~cm}$ ) and a $\mathrm{NaCl}$ crystal $(l=2 \mathrm{~cm}$ ). The laser input peak intensity had to be kept below $3 \times 10^{11} \mathrm{~W} / \mathrm{cm}^{2}$ for Infrasil and $\mathrm{NaCl}$ in order to avoid material damage. It should be emphasized that the intensity conversion is higher than the depicted energy conversion. Calculations show that approximately one fifth of the beam diameter and of the pulse duration contributes to parametric light generation at the onset of saturation $\left[I_{\mathrm{L}}(0) \simeq 5 \mathrm{X}\right.$ $\left.10^{10} \mathrm{~W} / \mathrm{cm}^{2}\right]$. At this relatively low input intensity, an intensity conversion of $2 \%$ is estimated for the center of the laser pulse.

3). Our substances $\mathrm{H}_{2} \mathrm{O}, \mathrm{D}_{2} \mathrm{O}$, Infrasil and $\mathrm{NaCl}$ possess three properties favorable for efficient four photon parametric light generation.

i). Their electronic absorption bands are in the

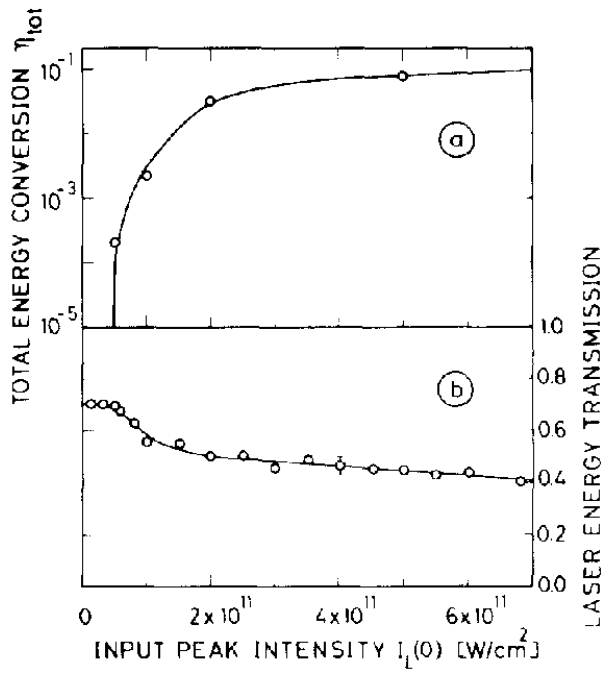

Fig. 2, a) Total energy conversion of laser light in to parametric light. Single picosecond light pulse of $\Delta t_{\mathrm{L}} \simeq 6 \mathrm{ps}, \lambda_{\mathrm{L}}=$ $1.06 \mu \mathrm{m}$ in water $(l=2 \mathrm{~cm})$. (b) Energy transmission of the same pulse versus input peak intensity.

vacuum ultraviolet. The dispersion of the refractive index and consequently the phase-mismatch are small in the visible and near ultraviolet. In addition, the loss of parametric light by linear (ultraviolet) absorption and by nonlinear two- and three-photon $\left(2 \nu_{\mathrm{L}}+\nu_{3}\right)$ absorption is not significant over most of the spectral range. On the contrary, in substances such as $\mathrm{CS}_{2}$, $\mathrm{C}_{6} \mathrm{H}_{5} \mathrm{NO}_{2}$ or $\mathrm{CH}_{2} \mathrm{I}_{2}$ with electronic absorption bands in the near ultraviolet $(\lambda \leqslant 4000 \AA)$, the parametric light generation was found to be drastically reduced even in the visible part of the spectrum $(\lambda \lesssim 6000 \AA)$.

ii). The gain coefficients for stimulated Raman scattering are very low for our materials $[6,7]$. The input intensity required for efficient Raman scattering is higher than the intensity value necessary for saturation of parametric light generation. In the $\mathrm{NaCl}$ crystal first-order Raman scattering is forbidden. For $\mathrm{H}_{2} \mathrm{O}, \mathrm{D}_{2} \mathrm{O}$ and Infrasil an energy conversion into first Stokes Raman light of $\lesssim 10^{-3}$ was measured in the saturation range for parametric four-photon interaction. In contrast, substances with high Raman gain showed considerable Raman conversion before para- 
metric light generation could be observed and the broadband light production was suppressed at higher intensities.

iii). The selected substances have low values of the nonlinear refractive index $n_{2}[5,8,9]$ where $n=n_{0}$ $+n_{2} E_{\mathrm{L}}^{2} / 2$. In the intensity range where our measurements were carried out $\left[I_{\mathrm{L}}(0) \leqslant 7 \times 10^{11} \mathrm{~W} / \mathrm{cm}^{2}\right.$ for $\mathrm{H}_{2} \mathrm{O}$ and $\mathrm{D}_{2} \mathrm{O} ; I_{\mathrm{L}}(0) \leqslant 3 \times 10^{11} \mathrm{~W} / \mathrm{cm}^{2}$ for Infrasil and $\mathrm{NaCl}$ ] self-focusing was not observed [10] . Self-focusing of the laser beam leads to severe disturbances in the light propagation, e.g. inhomogeneous intensity distribution in the beam cross-section and substantial beam divergence. In addition, the rapid intensity increase in part of the beam gives rise to material breakdown with optical absorption by free electrons and final destruction of the medium.

4). The parametric light generation in the infrared i.e. at the Stokes side of the pump frequency was investigated. It was found that the idler conversion efficiency $\eta_{4}$ decreases rapidly with frequency; it was substantially lower than the conversion efficiency $\eta_{3}$ at the equivalent signal frequency. For example, the energy conversion per wave number in the frequency range around $\widetilde{\nu}_{4}=4300 \mathrm{~cm}^{-1}$ is lower by a factor of approximately $10^{4}$ compared to the value at the signal frequency $\widetilde{v}_{3}=2 \widetilde{v}_{\mathrm{L}}-\widetilde{v}_{4}=14600 \mathrm{~cm}^{-1}$. For the parametric process $\omega_{\mathrm{L}}+\omega_{\mathrm{L}} \rightarrow \omega_{3}+\omega_{4}$ theory predicts $\eta_{4} / \eta_{3} \simeq \omega_{4} n_{3}^{3} / \omega_{3} n_{4}^{3}$, where $n_{4,3}$ is the index of refraction at the idler [4] and signal [3] frequency $(\omega=2 \pi \nu=2 \pi c \tilde{\nu})$. It is quite obvious that the simple equation does not explain our experimental observations. This fact is not surprising since saturation effects are not taken into account in deriving the equation. As soon as the laser light is depleted the parametric light generation rate is drastically reduced and the idler light, generated during the first part of the interaction length, suffers considerable linear absorption. This explanation is verified by numerical calculations (see below).

5 ). The energy transmission of the input pulse was measured in a sample of $\mathrm{H}_{2} \mathrm{O}(l=2 \mathrm{~cm})$; transmission values are plotted versus input peak intensity in fig. $2 \mathrm{~b}$. Similar results were obtained for $\mathrm{D}_{2} \mathrm{O}(l=2 \mathrm{~cm})$, Infrasil $(l=3 \mathrm{~cm})$ and $\mathrm{NaCl}(l=2 \mathrm{~cm})$. At low input intensities one starts with a transmission of $T=0.7$ in $\mathrm{H}_{2} \mathrm{O}$ due to the linear absorption at the laser frequency. For $I_{\mathrm{L}}(0)>5 \times 10^{10} \mathrm{~W} / \mathrm{cm}^{2}$ we find a nonlinear decrease of transmission. Comparing fig. $2 \mathrm{a}$ and fig. $2 b$ we see a larger decrease of laser transmission than expected from the total energy conversion. The nonlinear loss of laser light is most severe in the intensity range between $I_{\mathrm{L}}(0)=5 \times 10^{10} \mathrm{~W} / \mathrm{cm}^{2}$ and $1 \times 10^{11} \mathrm{~W} / \mathrm{cm}^{2}$. In this range the transmission reduces by approximately $\Delta T=12 \%$ while the total energy conversion increases to $\eta_{\text {tot }} \simeq 2 \times 10^{-3}$. We believe that the observed loss of laser light results from the infrared absorption of the parametrically generated idler light. The losses in the intensity range between $5 \times 10^{10}$ and $1 \times 10^{11} \mathrm{~W} / \mathrm{cm}^{2}$ are roughly estimated as follows: For parametric signal generation between 14000 and $19000 \mathrm{~cm}^{-1}$ the corresponding idler waves have frequencies below $5000 \mathrm{~cm}^{-1}$. In the latter region the infrared absorption of water is very large. Assuming that the idler light builds up to a total energy conversion of approximately $\eta_{4, \text { tot }}=5 \times 10^{-4}$ and that this value is retained over a length of $\Delta l \simeq 0.5 \mathrm{~cm}$ in spite of an average absorption coefficient of $\alpha_{4}$ $\simeq 500 \mathrm{~cm}^{-1}$, a loss of pump intensity of $\Delta I_{\mathrm{L}}$ $\simeq \alpha_{4} \Delta l \eta_{4} I_{\mathrm{L}}(0) \approx 0.13 I_{\mathrm{L}}(0)$ is estimated in agreement with the observed reduction of transmission.

6). Stimulated parametric four-photon interaction was calculated including pump depletion [11] and linear absorption at the signal and idler frequency. The primary parametric process $\omega_{L}+\omega_{L} \rightarrow \omega_{3}+\omega_{4}$ is considered here. The set of differential equations for this case is given in eqs. (1a)-(1c) [5]

$$
\begin{aligned}
& \frac{\partial E_{0}\left(\omega_{\mathrm{L}}\right)}{\partial z}=-\frac{1}{2} \alpha_{\mathrm{L}} E_{0}\left(\omega_{\mathrm{L}}\right)-\frac{\mathrm{i} 2 \pi \omega_{\mathrm{L}}}{n_{\mathrm{L}} c} E_{0}^{*}\left(\omega_{\mathrm{L}}\right) \\
& \times \int_{-\infty}^{\infty} \mathrm{d} \omega_{3} \int_{-\infty}^{\infty} \mathrm{d} \omega_{4} \chi^{(3)}\left(-\omega_{\mathrm{L}},-\omega_{\mathrm{L}}, \omega_{3}, \omega_{4}\right) \\
& \quad \times E_{0}\left(\omega_{3}\right) E_{0}\left(\omega_{4}\right) \exp (-\mathrm{i} \Delta k z), \\
& \frac{\partial E_{0}\left(\omega_{3}\right)}{\partial z}=-\frac{1}{2} \alpha_{3} E_{0}\left(\omega_{3}\right)-\frac{\mathrm{i} 2 \pi \omega_{3}}{n_{3} c} \\
& \quad \times \chi^{(3)}\left(-\omega_{3}, \omega_{\mathrm{L}}, \omega_{\mathrm{L}},-\omega_{4}\right) E_{0}^{*}\left(\omega_{4}\right) E_{0 \mathrm{~L}}^{2} \exp (\mathrm{i} \overline{\Delta k} z), \\
& \frac{\partial E_{0}^{*}\left(\omega_{4}\right)}{\partial z}=-\frac{1}{2} \alpha_{4} E_{0}^{*}\left(\omega_{4}\right)+\frac{\mathrm{i} 2 \pi \omega_{4}}{n_{4} c} \\
& \quad \times \chi^{(3) *}\left(-\omega_{4}, \omega_{\mathrm{L}}, \omega_{\mathrm{L}},-\omega_{3}\right) E_{0}\left(\omega_{3}\right) E_{0 \mathrm{~L}}^{* 2} \exp (-\mathrm{i} \overline{\Delta k z}) .
\end{aligned}
$$

$E_{0}\left(\omega_{\mathrm{L}}\right), E_{0}\left(\omega_{3}\right)$ and $E_{0}\left(\omega_{4}\right)$ are the complex amplitudes of the Fourier components of the electromagnetic field $E(t)=E_{\mathrm{L}}(t)+E_{3}(t)+E_{4}(t)$ at the fre- 
quencies $\omega_{\mathrm{L}}, \omega_{3}$ and $\omega_{4}$, respectively.

$E_{0 \mathrm{~L}}=\int_{-\infty}^{\infty} E_{0}\left(\omega_{\mathrm{L}}\right) \mathrm{d} \omega_{\mathrm{L}}=E_{\mathrm{L}}(t=0)$

is the peak amplitude of the input laser field. In refs. $[4,5]$ it is shown that

$\chi^{*}\left(-\omega_{4}, \omega_{L}, \omega_{L},-\omega_{3}\right) \sim \chi\left(-\omega_{3}, \omega_{L}, \omega_{L},-\omega_{4}\right)$.

In a similar way it can be shown that

$\chi\left(\omega_{L},-\omega_{L}, \omega_{3}, \omega_{4}\right) \simeq \chi^{*}\left(\omega_{3}, \omega_{L}, \omega_{L},-\omega_{4}\right)$.

In eqs. $(1 \mathrm{~b})$ and $(1 \mathrm{c})$ the integration over the frequency width of the input pulse around $\omega_{L}$ has been carried out. This integration is readily possible since the bandwidth of the pump pulse is small. The situation is different in eq. (1a) since the broad spectrum of parametrically generated light with different values of $E_{0}\left(\omega_{3}\right), E_{0}\left(\omega_{4}\right), \chi, \Delta k=k_{3}+k_{4}-2 k_{\mathrm{L}}, \alpha_{3}$ and $\alpha_{4}$ acts on the pump field. Furthermore, an integration over the beam cross-section would be recessary to calculate the energy conversion quantitatively. In order to come to practical solutions of eqs. (1a) to (1c) we simplified the problem as follows: Intensity conversions were calculated with values of $E_{0}\left(\omega_{3}\right), E_{0}\left(\omega_{4}\right)$, $\chi, \Delta k, \alpha_{3}$ and $\alpha_{4}$ assumed to be independent of frequency. Under these assumptions the integrations in eq. (la) could be readily carried out. Eqs. (1 a) to (lc) reduce to a set of four nonlinear or dinary differential equations by rewriting the complex quantities in amplitudes and phases and by separating the real and imaginary parts. One finally arrives at three amplitude equations and one phase difference equation [12]. The initial conditions for this set of equations were determined from the analytic solutions obtained for the case of no depletion [4,5]. In ligs. $3 a$ and $3 b$, numerical solutions of the normalized pump, signal and idler intensity are presented versus sample length for two sets of parameters. The two selected signal frequencies are $\widetilde{\nu}_{3}=10811 \mathrm{~cm}^{-1}$ (fig. $3 \mathrm{a}$ ), and $\widetilde{v}_{3}=17157 \mathrm{~cm}^{-1}$ (fig. $3 \mathrm{~b}$ ). A bandwidth of the signal light of $\Delta \widetilde{\nu}_{3}=1000 \mathrm{~cm}^{-1}$ was used and the field strengths $F_{0}\left(\omega_{3}\right)$ and $E_{0}\left(\omega_{4}\right)$ as well as the parameters $\alpha_{3}, \alpha_{4}, \chi$ and $\Delta k$ were assumed to be frequency independent within this frequency range. The linear absorption of the laser light $\left(\tilde{\nu}_{L}=\right.$ $9455 \mathrm{~cm}^{-1}$ ) is $\alpha_{\mathrm{L}}=0.15 \mathrm{~cm}^{-1}$ in water. The values of the parameters used in the calculations are listed in the figure caption. In fig. $3 a$ the idler absorption

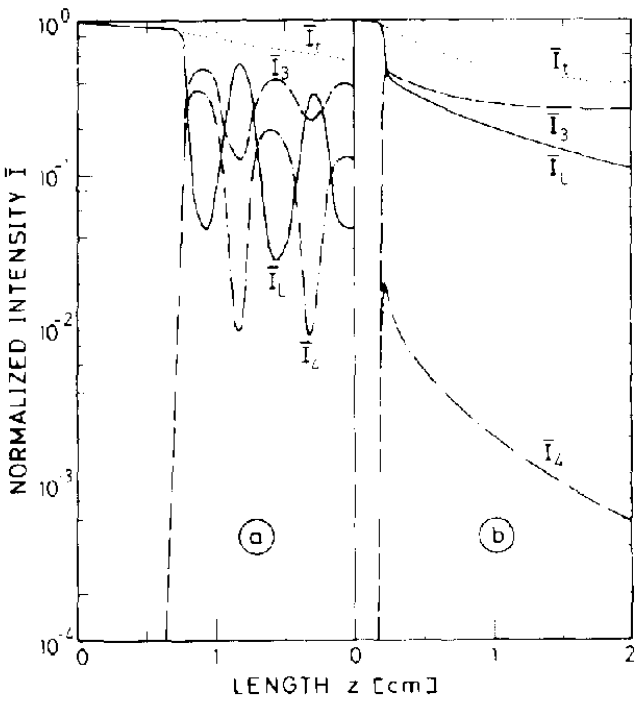

1'ig. 3. Calculated build-up of parametric light versus interaction length. Full line, normalized pump pulse intensity $\bar{I}_{L}=I_{\mathbf{L}}(z) / I_{\mathrm{L}}(0)$; dashed line, normalized signal intensity $I_{3}=I_{3}(z) / I_{L}(0)$; dash-dot line, normalized idler intensity $\vec{I}_{4}=I_{4}(z) / I_{\mathrm{L}}(0)$; dotted line normalized total intensity $\vec{I}_{1}$ $=\left[I_{\mathrm{L}}(z)+I_{3}(z)+I_{4}(z)\right] / I_{\mathrm{L}}(0) \cdot I_{\mathrm{L}}(0)=2 \times 10^{11} \mathrm{~W} / \mathrm{cm}^{2}$; $\alpha_{\mathrm{L}}=0.15 \mathrm{~cm}^{-1} ; I_{3}(0)$ and $I_{4}(0)$ start from quantum noise. Calculations were made over a signal frequency range of $1000 \mathrm{~cm}^{-1}$. (a) Low idler absorption and low phase-mismatch. The following data were used: $\widetilde{v}_{3}=10811 \mathrm{~cm}^{-1}$, $\alpha_{3}=0.09 \mathrm{~cm}^{-1}, \alpha_{4}=1.3 \mathrm{~cm}^{-1}, \Delta k=-3.3 \mathrm{~cm}^{-1}, \mathrm{x}^{(3)^{\prime}}$ $=8 \times 10^{-14}$ esu, $\chi^{(3)^{\prime \prime}}=3 \times 10^{-15}$ esu. (b) Strong idler absorption and zero phase-mismatch. The data are $\tilde{\nu}_{3}$ $=17160 \mathrm{~cm}^{-1}, \alpha_{3}=0.001 \mathrm{~cm}^{-1}, \alpha_{4}=400 \mathrm{~cm}^{-1}, \Delta k=0$, $x^{(3)}=.9 \times 10^{-13} \mathrm{esu}, x^{(3)^{\prime \prime}}=-8 \times 10^{-13} \mathrm{esu}$.

$\alpha_{4}=1.3 \mathrm{~cm}^{-1}$ is small at $\widetilde{\nu}_{4}=8100 \mathrm{~cm}^{-1}$. When the signal and idler intensity become larger than the depleted pump intensity and when the phase relations between the three waves are favorable, the signal and idler follow the reverse process $\omega_{3}+\omega_{4} \rightarrow \omega_{\mathrm{L}}+\omega_{\mathrm{I}}$. For increasing length of the sample an oscillatory behavior is calculated [12]. After a path of $2 \mathrm{~cm}$ the total light output $I_{\text {tot }}=I_{\mathrm{L}}(l)+I_{3}(l)+I_{4}(l)$ is reduced by $50 \%$ due to the small absorption at the laser and idler frequency. In fig. $3 b$ the idler absorption is higher, while the phase-mismatch is assumed to be zero $\left(\alpha_{4}=400 \mathrm{~cm}^{-1}, \Delta k=0\right)$. The highest conversion of laser light in to signal and idler light is obtained in an early part of the sample. For a longer sample length, the output of signal and idler is reduced due to laser light depletion and linear absorption. The 
total light output is reduced to $38 \%$. The curves depicted in Fig. 3 b show quite clearly that substantial laser depletion occurs with a signal intensity conversion of approximately $25 \%$. It should be noted that in the saturation range four-photon frequency conversion and higher order parametric four-photon processes take place which modify the curves of fig. 3.

7). The influence of self-phase modulation [1] of the pump pulse on the parametric four-photon light generation was investigated. A change of refractive index $\Delta n$ leads to a change of phase of the pump pulse $\phi_{\mathrm{L}}(z)=\omega_{\mathrm{L}} \Delta n z / c=\delta \mathrm{kz}$. The high electric field of the pump pulse generates a value of $\Delta n=n \cdots n_{0}$ $=\frac{1}{2} n_{2} E_{0 \mathrm{~L}}^{2}$. The change of phase can be accounted for by using an effective phase-mismatch $\Delta k_{\text {eff }}$ $=\Delta k \quad 2 \delta k$, where $\Delta k=k_{3}+k_{4}-2 k_{\mathrm{L}}$ is determined by the color dispersion of the medium. We have repeated our calculations including the phase change of the pump pulse resulting from the nonlinear coefficient $n_{2}:$ a value of $n_{2}=-2.5 \times 10^{-13}$ esu [5] was used corresponding to the experimental number for water. This value of $n_{2}$ leads to $\Delta n=-1.6 \times 10^{-4}$ and $\delta k=-9.5 \mathrm{~cm}^{-1}$ at $I_{\mathrm{L}}=2 \times 10^{11} \mathrm{~W} / \mathrm{cm}^{2}$. As a result we obtained curves which were qualitatively similar to those with $\delta k=0$. It should be noted that in our four-photon process we have, in general, a large phase-mismatch $\Delta k>\delta k$ over most of the frequency range [4]. Self-phase modulation reduces the parametric light generation for $\Delta k<\delta k$ [13].

In account of the self-phase modulation discussed here the frequency spectrum of the laser pulse $\left(I_{\mathrm{L}}\right.$ $\left.\simeq 5 \times 10^{11} \mathrm{~W} / \mathrm{cm}\right)$ is estimated to be broadened to $\Delta \tilde{\nu}_{\mathrm{L}} \simeq \frac{1}{2} \tilde{\nu}_{\mathrm{L}} n_{2} E_{0 \mathrm{~L}}^{2} / c \Delta t=90 \mathrm{~cm}^{-1}$ during the short pulse duration of $\Delta t=6 \times 10^{-12} \mathrm{~s}$.

In summary we would like to state: broad spectra with energy conversion of up to $10 \%$ can be generated in different substances several $\mathrm{cm}$ in length. The saturation of the conversion efficiency is qualitatively ac- counted for by the depletion of the laser intensity. Absorption at the idler frequency appears to be the major cause for the laser attenuation. The absence of self-focusing and the snall contribution of stimulated Raman scattering make $\mathrm{H}_{2} \mathrm{O}, \mathrm{D}_{2} \mathrm{O}, \mathrm{NaCl}$ and Infrasil glass very suitable materials for the generation of broadband picosecond pulses.

The authors are grateiul to Dr. A. Laubereau for valuable discussion and to $W$. Falkenstein for technical assistance.

\section{References}

[1] S.A. Akhmanov, R.V. Khokhlov and A.P. Sukhorukov, in: Laser Handbook, ed. by F.T. Arecchi and F.O. Schulz-DuBois (North-Holland, Amsterdam, 1972), ch. E3.

[2] N.N. Il'ichev, V.V. Korobkin, V.A. Korshunov, A.A. Malgutin, T.G. Okroaskvili and P.P. Pashinin, JLTPLetters 15 (1972) 133. W. Werncke, A. Lau, M. Pfeiffer, K. Lenz, H.-J. Weigmann and C.D. Thuy, Opt. Commun. 4 (1972) 413.

[3] G.F. Busch, R.P. Jones and P.M. Rentzepis, Chem. Phys. Letters 18 (1973) 178.

[4] A. Penzkofer, A. Laubcreau and W. Kaiser, Phys. Rev. Letters 31 (1973) 863.

[5] A. Penzkofer and W. Kaiser, to be published.

[6] O. Rahn, M. Maier and W. Kaiser, Opt. Commun. 1 (1969) 109.

[7] R.H. Stolen, F.P. Ippen, A.R. Tynes, Appl. Phys. Letters $20(1972) 62$.

[8] M. Paillette, Ann. Phys. (Paris) 4 (1969) 671.

[9] C.C. Wang, Phys. Rev. B2 (1970) 2045.

[10] A. Penzkofer, Opt. Commun. 11 (1974) 265.

[11] J. Frahm and R. Fischer, Annal. d. Physik 31 (1974) 143.

[12] J.A. Armstrong, N. Bloembergen, J. Ducuing and P.S. Pershan, Phys. Rev. 127 (1962) 1918.

[13] B. Crosignani, P.D. Porto, U. Ganiel, S. Solimento and A. Yariv, IFEE J. Quantum Electronics 8 (1972) 731. 